\title{
Elimination of Chrysanthemum stunt viroid (CSVd) from Meristem Tip Culture Combined with Prolonged Cold Treatment
}

\author{
Wina Dian Savitri1†, Kyung II Park ${ }^{2 \dagger}$, Su Min Jeon'1, Mi Young Chung ${ }^{3}$,Jeung-Sul Han ${ }^{4}$, and \\ Chang Kil Kim ${ }^{*}$ \\ 'Department of Horticultural Science, Kyungpook National University, Daegu 702-701, Korea \\ 2Department of Horticulture \& Life Science, Yeungnam University, Gyeongsan 712-749, Korea \\ ${ }_{3}^{3}$ Department of Agricultural Education, Sunchon National University, Sunchon 540-742, Korea \\ ${ }_{4}$ Department of Ecological Environment, Kyungpook National University, Sangju 742-711, Korea \\ ${ }^{*}$ Corresponding author: ckkim@knu.ac.kr \\ †These authors contributed equally to this work.
}

Received September 21, 2012 / Revised February 13, 2013 / Accepted February 23, 2013

CKorean Society for Horticultural Science and Springer 2013

Abstract. Chrysanthemum production in Korea has recently been greatly affected by the spread of Chrysanthemum stunt viroid (CSVd) infection, necessitating the use of CSVd-free stocks to ensure successful chrysanthemum cultivation. We investigated the effects of low temperature $\left(4^{\circ} \mathrm{C}\right)$, antiviral chemicals (ribavirin and amantadine) and a combination of these treatments on CSVd elimination by meristem tip cultures using plantlets that originated from CSVd-infected chrysanthemum 'Ency'. Neither antiviral agents led to CSVd elimination, despite the suppression of meristem tip growth in a concentration dependent manner. However, the CSVd elimination rate increased up to $42.8 \%$ when meristem tips were excised after storage at $4^{\circ} \mathrm{C}$ for two months. The most effective results were obtained from a combination of low temperature for three months at $4^{\circ} \mathrm{C}$ followed by meristem tip culture on media containing 50 and $100 \mathrm{mgL}^{-1}$ ribavirin. These results suggest that antiviral agents can also be useful for CSVd elimination if their treatment is combined with prolonged periods of low temperature. This is the first report of eradication of viroids from spray type chrysanthemum bred in Korea.

Additional key words: amantadine, CSVd-free, low temperature, nested PCR, ribavirin

Hort. Environ. Biotechnol. 54(2):177-182. 2013.

DOI 10.1007/s13580-013-0141-8 
